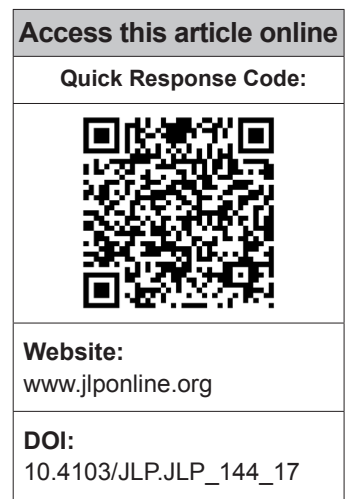

Department of Obstetrics and Gynecology, Selcuk University, ${ }^{1}$ Department of Obstetrics and

Gynecology, Konya Training and Research

Hospital, ${ }^{2}$ Department of Biochemistry, Selçuk University, Konya, Turkey

Address for correspondence: Dr. Ersin Çintesun, Department of Obstetrics and Gynecology,

Selcuk University, Konya, Turkey. E-mail: ersincintesun@ gmail.com

Submission: 12-09-2017 Accepted: 28-11-2017

\title{
Systemic inflammatory response markers in preeclampsia
}

\author{
Ersin Çintesun, Feyza Nur Incesu Çintesun'1, Huriye Ezveci, Fikret Akyürek², \\ Çetin Çelik
}

\section{Abstract:}

PURPOSE: Neutrophil-lymphocyte ratio (NLR), platelet-lymphocyte ratio (PLR), platelet distribution width (PDW), red cell distribution width (RDW), mean platelet volume (MPV), and plateletcrit (PCT) are known as systemic inflammatory response markers. In this study, we aimed to evaluate changes in NLR, PLR, PDW, RDW, MPV, and PCT in preeclampsia (PE) and their use in predicting its severity.

MATERIALS AND METHODS: This is a retrospective case-control study. The study comprised 64 control group (healthy pregnant females), 51 females with mild PE, and 13 with severe PE. These three groups were compared with demographic data and inflammation markers.

RESULTS: There were no statistically significant differences between healthy pregnant females and preeclaptic females in terms of median age, hemoglobin, lymphocyte, neutrophil, platelet, NLR, PLR, PDV, RDW, MPV, PCT $(P>0.05)$. The control group has a higher number of gravity and parity than the PE group $(P<0.001)$. MPV value is a lower PE group than the control group $(P<0.001)$. Both gravity and parity were significantly higher in the patients with mild PE than in the control group $(P<0.001)$. MPV value was statistically higher in the control group compared both mild and severe PE $(P<0.001)$, however, no statistical difference between mild and severe PE $(P=0.305)$.

CONCLUSIONS: MPV may be clinically useful markers in the prediction of PE. Further, prospective multicenter studies are needed to reveal the association between these markers and PE.

Key words:

Inflammatory, lymphocyte, neutrophil, platelet, preeclampsia

\section{Introduction}

Dreeclampsia (PE) is characterized by a new onset of hypertension after 20 weeks of gestation in a previously normotensive pregnant woman accompying with either proteinuria or end-organ dysfunction. $\mathrm{PE}$ is one of the causes of maternal-fetal morbidity and mortality, affecting approximately $4.6 \%$ of all pregnancies worldwide. ${ }^{[1]}$ Cause of PE is not known definitively, but it is likely to be related with inflamatuar process. Studies have shown that hyper-reactivation of inflammatory cells and immunologic responses of neutrophils and lymphocytes take place by releasing inflammatory cytokines and autoantibodies resulting to endothelial dysfunction. ${ }^{[2]}$

This is an open access journal, and articles are distributed under the terms of the Creative Commons Attribution-NonCommercial-ShareAlike 4.0 License, which allows others to remix, tweak, and build upon the work non-commercially, as long as appropriate credit is given and the new creations are licensed under the identical terms.

For reprints contact: reprints@medknow.com
Clinical phenotypes of PE can be defined as clinical responses to generalized endothelial dysfunction which causes vasoconstriction and end-organ ischemia. ${ }^{[3,4]}$

The changes in the values of hematological parameters including, neutrophil-lymphocyte ratio (NLR), platelet-lymphocyte ratio (PLR), platelet distribution width (PDW), red cell distribution width (RDW), mean platelet volume (MPV), and plateletcrit (PCT) are known as systemic inflammatory response markers. ${ }^{[5]}$ Although these markers have been investigated in patients with PE at previous studies, the results were conflicting. ${ }^{[6-10]}$

In this study, we aimed to evaluate the relationship between PE and changes in NLR, PLR, PDW, RDW, MPV, and PCT and their

How to cite this article: ÇintesunE, Incesu Çintesun FN, Ezveci $\mathrm{H}$, Akyürek F, Çelik Ç. Systemic inflammatory response markers in preeclampsia. J Lab Physicians 2018;10:316-9. 
use in predicing PE severity. We aimed to evaluate all the parameters in the same study while these parameters were generally working separately in other studies.

\section{Materials and Methods}

This is a retrospective case-control study conducted at the Department of Obstetrics and Gynecology a University Hospital, between January 1, 2016, and May 1, 2017. The local ethics committee approved the study. This study has 130 patients. Sixty-six patients were in the control group and sixty-four patients were in the PE group. PE group has 51 mild PE and 13 severe PE patients. The control group was randomly selected from patients without PE and systemic disease, who were the third trimester of the patients followed up and treated in our hospital. Diagnosing criteria of PE was based on criteria of the American College of Obstetrics and Gynecology in 2013. ${ }^{[1]}$ They were: Blood pressure $\geq 140 \mathrm{mmHg}$ systolic or $90 \mathrm{mmHg}$ diastolic on two occasions at least $4 \mathrm{~h}$ apart after 20 weeks of gestation with a previously normal blood pressure or blood pressure $\geq 160 \mathrm{mmHg}$ systolic or $\geq 110 \mathrm{mmHg}$ diastolic and proteinuria (dipstick reading of $1+$, protein/creatinine ratio $\geq 0.3$, or $\geq 0.3 \mathrm{~g}$ in a $24 \mathrm{~h}$ urine specimen) or in the absence of proteinuria, new-onset hypertension with the new onset of any of the following: thrombocytopenia (Platelet count $\leq 100,000 / \mathrm{mlt}$ ), renal insufficiency serum creatinine concentrations $\geq 1.1 \mathrm{mg} / \mathrm{dl}$, elevated blood concentrations of liver transaminases to twice normal concentration, pulmonary edema, and cerebral visual symptoms.

Diagnosing criteria of severePEwerebased on:symptoms of central nervous system dysfunction (new-onset cerebral or visual disturbance), hepatic abnormality (severe persistent right upper quadrant or epigastric pain unresponsive to medication serum transaminase concentration $\geq 2$ times the upper limit of the normal range, or both), severe blood pressure elevation (systolic blood pressure $\geq 160 \mathrm{mmHg}$ or diastolic blood pressure $\geq 110 \mathrm{mmHg}$ on two occasions at least $4 \mathrm{~h}$ apart while the patient is on bedrest), thrombocytopenia ( $<100,000$ platelets $/$ microL), renal abnormality (serum creatinine $>1.1 \mathrm{mg} / \mathrm{dL}$ ), and pulmonary edema. ${ }^{[11]}$

Patients who have chronic systemic disease during pregnancy such as diabetes mellitus, nephropathy, renal or hepatic dysfunction, active local or systemic infection, chorioamnionitis, or any medication such as corticosteroids were excluded to this study. Patient with any signs and symptoms of active infection (pain, fever, or vaginal discharge), were excluded from the study. Serum samples for complete blood count with differentials including neutrophil leukocyte, platelets, NLR, PNR, RDW, MPV, PCT, and PDW were obtained at the admission of the patients to the emergency department, before the initiation of any medical treatment. Venous blood samples were kept in ethylenediaminetetraacetic acid samples tubes $(5 \mathrm{~mL})$, and processed within $2 \mathrm{~h}$, using an automatic blood cell counter.

Data analysis was performed using SPSS version 22 (SPSS Inc., Chicago, IL, USA). The Kolmogorov-Smirnov test and histogram were used to analyze the distribution of thedata. Variablesaregiven as mean (minimum-maximum) or median (minimum-maximum). Nonparametric tests were performed to compare data within between two groups (Mann-Whitney U-test) and within three groups (Kruskal-Wallis test). Parametric tests were performed to compare data within between two groups (Independent-Simple $t$-test) and within three groups (One-way ANOVA test) in PE group, Tukey and Tamhane's T2 tests were used for multiple comparisons.

\section{Results}

Comparison of demographic features, subclinical inflammatory marker levels between control groups and PEgroup were shown in Table1. There were no statistically significant differences between the two groups in terms of median age, hemoglobine, lymphocyte, neutrophil, platelet, NLR, PLR, PDV, RDW, MPV, PCT $(P>0.05)$. Control group had a higher number of gravity and parity than the PE group $(P<0.001)$. MPV value was a lower PE group than the control group $(P<0.001)$.

The comparison of subclinical inflammatory marker levels among three groups is shown in Table 2. Both gravity and parity were higher in the patients with mild PE than in the control group, and these differences showed a statistically significant $(P<0.001)$. MPV value was statistically higher in the control group compared both mild and severe PE $(P<0.001)$, however, no statistical difference between mild and severe PE $(P=0.305)$. No statistically significant difference was observed between the groups when compared to the other data $(P>0.05)$.

\section{Discussion}

Preeklampsia is a multisystemic hypertensive disorder and its etiology is not defined clearly. Investigations showed several factors including activation of inflammatory cells and immunologic responses in which neutrophils, lymphocytes, and thrombocytes participate by releasing inflammatory cytokines and autoantibodies. ${ }^{[2,10,12]}$ Recent studies showed and suggested that systemic inflammatory markers, obtained from complete blood count in peripheral blood, such as NLR, PLR, RDW, MPV, and PCT have prognostic and predictive values in various diseases including coronary artery disease, inflammatory diseases, otoimmun disease, PE and gynecologic, or gastrointestinal malignancies. ${ }^{[10,12,13]}$ 
Table 1: Comparison of demographic features, subclinical inflammation marker levels between control groups and preeclampsia group

\begin{tabular}{lccc}
\hline & Control group $(n=66)$ & Preeclampsia $(n=64)$ & $P$ (within 2 groups) \\
\hline Age & $29(17-40)$ & $28(17-41)$ & $0.397^{*}$ \\
Gravida^ & $3(1-5)$ & $2(1-5)$ & $<0.001^{* *}$ \\
Parity^ & $2(0-3)$ & $1(0-4)$ & $<0.001^{* *}$ \\
Hemoglobine & $12.1(8.8-16.0)$ & $11.7(8.6-15.8)$ & $0.125^{*}$ \\
Lymphocyte & $1.97(0.43-3.32)$ & $1.98(0.49-6.12)$ & $0.949^{*}$ \\
Neutrophil & $7.74(3.93-24.54)$ & $8.72(2.9-21.1)$ & $0.80^{* *}$ \\
Platelet & $212(115-397)$ & $223(101-463)$ & $0.376^{*}$ \\
NLR & $4.40(1.81-22.72)$ & $5.49(1.21-23.22)$ & $0.200^{* *}$ \\
PLR & $118.05(48.54-553.49)$ & $129.26(47.39-460.27)$ & $0.228^{* *}$ \\
PDW & $17.58(16.30-19.30)$ & $17.86(16.1-21.0)$ & $0.136^{* *}$ \\
RDW & $15.53(12.1-26.1)$ & $15.18(12.0-28.0)$ & $0.774^{* *}$ \\
MPV & $8.85(6.5-12.7)$ & $7.82(5.23-10.7)$ & $<0.001^{*}$ \\
PCT & $0.18(0.08-0.34)$ & $0.18(0.08-0.35)$ & $0.966^{*}$
\end{tabular}

Values are given as median (minimum-maximum); others given as mean (minimum-maximum). ${ }^{*}$ Independent simple $\mathrm{t}$ test. ${ }^{* *}$ Mann-Whitney $\mathrm{U}$ test. $\mathrm{NLR}=$ Neutrophil lymphocyte ratio, $\mathrm{PLR}=$ Platelet lymphocyte ratio, RDW $=$ Red cell distribution width, MPV $=$ Mean platelet volume, $\mathrm{PCT}=\mathrm{Plateletcrit}$, PDW = Platelet distribution width

Table 2: Comparison of subclinical inflammatory marker levels among three groups

\begin{tabular}{|c|c|c|c|c|c|c|c|}
\hline & Control group $(n=66)$ & $\begin{array}{l}\text { Mild preeclampsia } \\
\qquad(n=51)\end{array}$ & $\begin{array}{l}\text { Severe preeclampsia } \\
\qquad(n=13)\end{array}$ & $\begin{array}{c}P \text { (within } 3 \\
\text { groups) }\end{array}$ & $\begin{array}{l}P \text { (control vs. } \\
\text { mild PE) }\end{array}$ & $\begin{array}{l}P \text { (control vs. } \\
\text { severe PE) }\end{array}$ & $\begin{array}{l}P \text { (mild vs. } \\
\text { severe PE) }\end{array}$ \\
\hline Age & $29(17-40)$ & $28(17-41)$ & $28(18-38)$ & $0.699^{*}$ & $0.719^{*}$ & $0.851^{*}$ & $0.998^{*}$ \\
\hline Gravida^ & $3(1-5)$ & $2(1-5)$ & $2(1-5)$ & $<0.001^{* *}$ & $<0.001^{* *}$ & $0.478^{* *}$ & $0.824^{* *}$ \\
\hline Parity^ & $2(0-3)$ & $1(0-4)$ & $1(0-3)$ & $<0.001^{* *}$ & $<0.001^{* *}$ & $0.071^{\star *}$ & $0.90^{* *}$ \\
\hline Hemoglobine & $12.1(8.8-16.0)$ & $11.7(8.6-14.9)$ & $11.8(9.9-15.8)$ & $0.298^{*}$ & $0.276^{*}$ & $0.783^{*}$ & $0.96^{*}$ \\
\hline Lymphocyte & $1.97(0.43-3.32)$ & $1.99(0.49-6.12)$ & $1.91(0.73-3.54)$ & $0.938^{*}$ & $0.982^{*}$ & $0.966^{*}$ & $0.934^{*}$ \\
\hline Neutrophil & $7.74(3.93-24.54)$ & $8.79(2.90-21.10)$ & $8.48(3.13-18.90)$ & $0.171^{* *}$ & $0.236^{* *}$ & $0.760^{* *}$ & $0.955^{\star *}$ \\
\hline Platelet & $212(115-397)$ & $220(103-343)$ & $236(101-463)$ & $0.489^{*}$ & $0.841^{*}$ & $0.472^{*}$ & $0.698^{*}$ \\
\hline NLR & $4.40(1.81-22.72)$ & $5.37(1.21-23.22)$ & $5.94(1.31-18.18)$ & $0.395^{++}$ & $0.422^{++}$ & $0.724^{++}$ & $0.982^{++}$ \\
\hline PLR & $118.05(48.54-553.49)$ & $123.95(47.39-391.04)$ & $150.08(69.06-460.27)$ & $0.471^{* *}$ & $0.891^{* *}$ & $0.279^{* *}$ & $0.442^{* *}$ \\
\hline PDW & $17.58(16.30-19.30)$ & $17.8(16.1-21.0)$ & $17.9(16.8-19.2)$ & $0.168^{++}$ & $0.398^{++}$ & $0.204^{++}$ & $0.975^{++}$ \\
\hline RDW & $15.53(12.1-26.1)$ & $15.03(12.0-28.0)$ & $15.7(13.8-20.2)$ & $0.298^{* *}$ & $0.60^{* *}$ & $0.96^{\star *}$ & $0.68^{* *}$ \\
\hline MPV & $8.85(6.5-12.7)$ & $7.91(6.02-10.7)$ & $7.46(5.23-8.40)$ & $<0.001^{+}$ & $<0.001^{+}$ & $<0.001^{+}$ & $0.305^{+}$ \\
\hline PCT & $0.18(0.08-0.34)$ & $0.18(0.08-0.29)$ & $0.18(0.08-0.35)$ & $0.916^{*}$ & $0.994^{*}$ & $0.929^{*}$ & 0.909 \\
\hline
\end{tabular}

Values are given as median (minimum-maximum); others given as mean (minimum-maximum). ${ }^{\star}$ One-way ANOVA Tukey HSD, **Kruskall-Wallis test Tukey HSD, + One-way ANOVA Tamhane post hoc, ${ }^{+}$Kruskall-Wallis test Tamhane post hoc. NLR $=$Neutrophil-lymphocyte ratio, PLR $=$Platelet-lymphocyte ratio, $\mathrm{RDW}=$ Red cell distribution width, MPV $=$ Mean platelet volume, $\mathrm{PCT}=$ Plateletcrit, $\mathrm{PE}=$ Preeclampsia, $\mathrm{PDW}=\mathrm{Platelet}$ distribution width, $\mathrm{ANOVA}=\mathrm{Analysis}$ of variance, $\mathrm{HSD}=$ Honestly significant difference

Activated leukocytes are responsible for the vascular dysfunction associated with preeclampsia. ${ }^{[14,15]}$ NLR and PLR markers were thought to predict the presence and severity of PE while conflicting results have been reported in the literature. Yücel et al. found that PLR was significantly lower in the patients with severe $\mathrm{PE}$ than in the control group, however, there was no significant differences in NLR between the groups. ${ }^{[13]}$ Gezer et al. showed that high levels of NLR and PLR during the first trimester were independent predictors of preeclampsia. ${ }^{[16]}$ Kirbas et al. also found that NLR values were significantly higher in the severe PE group than control group, and PLR values were found to be significantly higher in the patients with severe PE compared to the patients with mild preeclampsia. ${ }^{[17]}$ In this study, NLR and PLR levels are not significant differences between groups.
PCT and MPV show the variation of platelet volume are thought to be the markers of platelet activation. PCT is calculated by platelet count multiply with MPV and divided to $10,000 \cdot{ }^{[18,19]}$ It is known that platelets become larger due to hypertension, but it is not clear how PE influences the platelets diameters. ${ }^{[8]}$ Yücel et al. found that PCT and MPV were associated with the presence of PE, and grouping patients with severe PE from mild PE group. ${ }^{[13]}$ Karateke et al. found that PCT level was significantly lower in the PE group as compared to control group, and PDW and MPV levels were significantly higher in severe preeclamptic patients than mild preeclamptic patients. ${ }^{[14]}$ In our study, PCT level did not differ between groups, severe PE and healthy pregnant women. MPV level was statistically higher in the control group compared both mild and severe PE $(P<0.001)$, however, no statistical difference were found between mild and severe PE groups $(P=0.305)$. In 
addition, area under the curve was insufficient to predict the presence of PE.

The RDW shows a variation in erythrocyte volume called as anisocytosis. High-RDW levels are believed to reflect increased inflammation, but this mechanism is not known. ${ }^{[15]}$ The relationship between RDW and PE was investigated in several studies: Yücel et al. found that RDW was significantly higher in severe preeclamptic pregnants compared to the controls. ${ }^{[13]}$ Abdullahi et al. found that RDW was not correlated with PE among pregnant Sudanese women. ${ }^{[8]}$ Y1lmaz et al. found that RDW values were significantly higher in PE group than control group and RDW levels were significantly higher in severe PE group than mild PE group in subgroup analyses. ${ }^{[20]}$ In our study, RDW level did not differ between patients.

Limitations of this study are its retrospective design, being a single-center study and the relatively small population in the severe PE group. Moreover, we do not know about patient's iron and folic acid usage or their blood levels.

To summarize, there are conflicting data in the literature about the effectiveness of NLR, PLR, RDW, PDV, and PCT in predicting presence and severity of PE. $[8,13,14,21]$ According to the results of our study: NLR, PLR, RDW, PCT, and PDW levels did not differ between severe preeclamptic, mild preeclamptic, and healthy pregnant women. Interestingly, in our study, the MPV levels were found significantly lower in the PE group than in the control group and MPV levels were lower in severe preeclamptic patients than in mild preeclamptic patients.

\section{Conclusions}

These markers are routinely obtained on laboratory evaluation of complete blood count, making this a potentially cost-effective, inexpensive predictive tool for assessing preeclampsia in a clinical setting, but there are many conflictions about the use of PE in the literature. Further, prospective multicenter studies are needed to reveal the association between these markers and PE.

\section{Financial support and sponsorship Nil.}

\section{Conflicts of interest}

There are no conflicts of interest.

\section{References}

1. Abalos E, Cuesta C, Grosso AL, Chou D, Say L. Global and regional estimates of preeclampsia and eclampsia: A systematic review. Eur J Obstet Gynecol Reprod Biol 2013;170:1-7.

2. Laresgoiti-Servitje E, Gómez-López N, Olson DM. An immunological insight into the origins of pre-eclampsia. Hum Reprod Update 2010;16:510-24.
3. Redman CW, Sacks GP, Sargent IL. Preeclampsia: An excessive maternal inflammatory response to pregnancy. Am J Obstet Gynecol 1999;180:499-506.

4. Powe CE, Levine RJ, Karumanchi SA. Preeclampsia, a disease of the maternal endothelium: The role of antiangiogenic factors and implications for later cardiovascular disease. Circulation 2011;123:2856-69.

5. Zhang WW, Liu KJ, Hu GL, Liang WJ. Preoperative platelet/lymphocyte ratio is a superior prognostic factor compared to other systemic inflammatory response markers in ovarian cancer patients. Tumour Biol 2015;36:8831-7.

6. Han L, Liu X, Li H, Zou J, Yang Z, Han J, et al. Blood coagulation parameters and platelet indices: Changes in normal and preeclamptic pregnancies and predictive values for preeclampsia. PLoS One 2014;9:114488.

7. Kurt RK, Aras Z, Silfeler DB, Kunt C, Islimye M, Kosar O, et al. Relationship of red cell distribution width with the presence and severity of preeclampsia. Clin Appl Thromb Hemost 2015;21:128-31.

8. Abdullahi H, Osman A, Rayis DA, Gasim GI, Imam AM, Adam I, et al. Red blood cell distribution width is not correlated with preeclampsia among pregnant Sudanese women. Diagn Pathol 2014;9:29.

9. Kashanian M, Hajjaran M, Khatami E, Sheikhansari N. Evaluation of the value of the first and third trimester maternal mean platelet volume (MPV) for prediction of pre-eclampsia. Pregnancy Hypertens 2013;3:222-6.

10. Vilchez G, Lagos M, Kumar K, Argoti P. Is mean platelet volume a better biomarker in pre-eclampsia? J Obstet Gynaecol Res 2017;43:982-90.

11. American College of Obstetricians and Gynecologists, Task Force on Hypertension in Pregnancy. Hypertension in pregnancy. Report of the American college of obstetricians and gynecologists' task force on hypertension in pregnancy. Obstet Gynecol 2013;122:1122-31.

12. Vilchez G, Lagos M, Kumar K, Argoti P. Is mean platelet volume a better biomarker in pre-eclampsia? The journal of obstetrics and gynaecology research 2017;43:982-90.

13. Yücel B, Ustun B. Neutrophil to lymphocyte ratio, platelet to lymphocyte ratio, mean platelet volume, red cell distribution width and plateletcrit in preeclampsia. Pregnancy Hypertens 2017;7:29-32.

14. Karateke A, Kurt RK, Baloğlu A. Relation of platelet distribution width (PDW) and platelet crit (PCT) to preeclampsia. Ginekol Pol 2015;86:372-5.

15. Wen Y. High red blood cell distribution width is closely associated with risk of carotid artery atherosclerosis in patients with hypertension. Exp Clin Cardiol 2010;15:37-40.

16. Gezer C, Ekin A, Ertas IE, Ozeren M, Solmaz U, Mat E, et al. High first-trimester neutrophil-to-lymphocyte and platelet-to-lymphocyte ratios are indicators for early diagnosis of preeclampsia. Ginekol Pol 2016;87:431-5.

17. Kirbas A, Ersoy AO, Daglar K, Dikici T, Biberoglu EH, Kirbas O, et al. Prediction of preeclampsia by first trimester combined test and simple complete blood count parameters. J Clin Diagn Res 2015;9:20-3.

18. Giacomini A, Legovini P, Gessoni G, Antico F, Valverde S, Salvadego MM, et al. Platelet count and parameters determined by the bayer ADVIA 120 in reference subjects and patients. Clin Lab Haematol 2001;23:181-6.

19. Adibi P, Faghih Imani E, Talaei M, Ghanei M. Population-based platelet reference values for an Iranian population. Int J Lab Hematol 2007;29:195-9.

20. Yılmaz ZV, Yılmaz E, Küçüközkan T. Red blood cell distribution width: A simple parameter in preeclampsia. Pregnancy Hypertens 2016;6:285-7.

21. Serin S, Avcı F, Ercan O, Köstü B, Bakacak M, Kıran H, et al. Is neutrophil/lymphocyte ratio a useful marker to predict the severity of pre-eclampsia? Pregnancy Hypertens 2016;6:22-5. 